\title{
Assembly of proteasome subunits into non-canonical complexes in vivo
}

\author{
Lindsay J. Hammack and Andrew R. Kusmierczyk* \\ Department of Biology \\ Indiana University-Purdue University Indianapolis \\ 723 West Michigan Street \\ Indianapolis, IN \\ 46202 \\ United States of America
}

*corresponding author: akusmier@iupui.edu

This is the author's manuscript of the article published in final edited form as:

Hammack, L. J., \& Kusmierczyk, A. R. (2017). Assembly of proteasome subunits into non-canonical complexes in vivo. Biochemical and Biophysical Research Communications, 482(1), 164-169.

https://doi.org/10.1016/j.bbrc.2016.11.024 


\begin{abstract}
Proteasomes exist in all domains of life. In general, they are comprised of a compartmentalized protease whose activity is modulated by one or more regulatory complexes with which it interacts. The quaternary structure of this compartmentalized protease, called the $20 \mathrm{~S}$ proteasome, is absolutely conserved and consists of four heptameric rings stacked coaxially. The rings are made of structurally related $\alpha$ and $\beta$ subunits. In eukaryotes, assembly factors chaperone the $\alpha$ and $\beta$ subunits during 20S biogenesis. Here we demonstrate that proteasome subunits can assemble into structures other than the canonical 20 s proteasome in vivo. Specifically, the yeast $\alpha 4$ subunit forms high molecular weight complexes whose abundance increases when proteasome function is compromised. Results from a disulfide crosslinking approach are consistent with these complexes being ring-shaped. Though several eukaryotic $\alpha$ subunits can form rings when expressed recombinantly in bacteria, this is the first evidence that such non-canonical complexes exist in vivo.
\end{abstract}

\title{
Key words
}

Proteasome, assembly, yeast, protein complex, native PAGE

\section{Abbreviations}

$\mathrm{CP}$, core particle; HMWC, high molecular weight complex; RP, regulatory particle; ICAR, immobilized-cobalt affinity resin 


\section{Introduction}

The $26 \mathrm{~S}$ proteasome comprises two major sub assemblies: the $20 \mathrm{~S}$ proteasome, also known as the core particle (CP), and a 19S regulatory particle (RP) $[1,2]$. The CP consists of four heptameric rings built up from two structurally related subunits, $\alpha$ and $\beta$, in an $\alpha_{7} \beta_{7} \beta_{7} \alpha_{7}$ arrangement $[3,4,5]$. In eukaryotes, the $\alpha$ and $\beta$ rings contain seven different subunits, and peptide hydrolysis activity resides within the $\beta 1, \beta 2$, and $\beta 5$ subunits. Assembly of the CP is guided by structural features intrinsic to the CP subunits and by extrinsic factors in the form of dedicated assembly factors [6]. There are five such factors in eukaryotes, Ump1 and Pba1-4 (called PAC1-4 in mammals).

Assembly of the CP begins with the formation of $\alpha$-rings which serve as a platform for the subsequent entry of $\beta$ subunits until a half-proteasome (i.e. $\alpha_{7} \beta_{7}$ ) intermediate is formed [7]. Two half-proteasomes dimerize to give rise to the $\mathrm{CP}$, a process that coincides with the autocatalytic removal of propeptides on the enzymatically active $\beta$ subunits [8]. The assembly factors carry out several functions, including: ensuring the correct placement of subunits within the rings [9], performing key checkpoint functions [10], preventing non-desirable interactions between CP subunits themselves $[11,12]$ and preventing binding of RP to immature CP species [13]. The end result is a fully functional CP with all subunits occupying a defined position. However, evidence has been mounting that the structure of the CP need not be considered fixed. In the yeast Saccharomyces cerevisiae, the $\alpha 3$ subunit is not essential for viability; in $\alpha 3 \Delta$ yeast cells, an alternative CP is formed in which a second copy of $\alpha 4$ occupies the position normally held by $\alpha 3$ [14]. These " $\alpha 4-\alpha 4$ proteasomes", so-called because their $\alpha$-rings now contain two neighboring $\alpha 4$ subunits, also arise in yeast when the Pba3-Pba4 assembly factor is absent [9] even though $\alpha 3$ is still present. Recently, $\alpha 4-\alpha 4$ proteasomes were shown to exist in mammalian cells, arguing that formation of this alternative CP is evolutionarily conserved [15]. Here, we present additional evidence that the CP structure need not be considered 
immutable. In addition to enabling the formation of $\alpha 4-\alpha 4$ proteasomes, we find that $\alpha 4$ assembles into high molecular weight complexes (HMWCs) that are most likely $\alpha 4$ rings. Some eukaryotic $\alpha$ subunits can form non-canonical rings when expressed recombinantly in bacteria but the relevance of these structures was not clear $[16,17,18,19]$. This is the first report of such structures existing in wild-type cells in vivo, raising the possibility of functional significance.

\section{Materials and Methods}

\subsection{Strains and Yeast Culture}

Yeast strains are listed in Supplementary Table 1. The $\alpha 2 \mathrm{HF}$ strain (MATa) was generated by backcrossing the $\alpha 2 \mathrm{HF}$ MAT $\alpha$ strain kindly provided by Mark Hochstrasser. The crosslinkable $\alpha 4$ ( $\alpha 4 C C)$ was engineered as described [14], except we employed a C-terminal Flag tag. One liter yeast cultures were grown in YPD at $30{ }^{\circ} \mathrm{C}$ to mid-log phase. Yeast cells were harvested by centrifugation at $4,000 \times g$ and the pellets washed with $40 \mathrm{ml}$ of $\mathrm{H}_{2} \mathrm{O}$ prior to storage at -80 ${ }^{\circ} \mathrm{C}$.

\subsection{Yeast Lysis and Flag Purification}

Yeast cell pellets were thawed in cold water and gently and resuspended in $48 \mathrm{ml}$ of Buffer A (40 mM Hepes-NaOH pH 7.5, 10\% (v/v) glycerol, 350 mM NaCl, 0.1\% (v/v) Tween-20 supplemented with yeast protease inhibitors (Sigma) according to manufacturer's instructions). The suspension was transferred to a $50 \mathrm{ml}$ bead beater lysis chamber along with $0.5 \mathrm{~mm}$ glass beads (BioSpec). The yeast cells were lysed via bead beating for 15 cycles (1 min beating followed by $1 \mathrm{~min}$ rest/cooling). Total lysates were centrifuged at $11,000 \mathrm{rpm}$ for 1 hour at $4{ }^{\circ} \mathrm{C}$ in a Beckman J2-21M centrifuge using a JA-20 rotor. The supernatant (soluble lysate) was transferred to a fresh $50 \mathrm{ml}$ conical tube and the protein concentration measured via Bradford Assay or BCA Protein Assay Kit (ThermoScientific). When processing parallel samples, equal amounts of protein from the soluble lysate were incubated with $200 \mu$ of anti-Flag agarose resin 
(Sigma) overnight at $4{ }^{\circ} \mathrm{C}$. The resin was collected in a $30 \mathrm{ml}$ gravity column (Bio-Rad), washed with $60 \mathrm{ml}$ of Buffer $\mathrm{A}$, transferred to a fresh microcentrifuge tube, and centrifuged for 30 seconds at 4,000 $\times g$. Excess Buffer A was aspirated, and the Flag-tagged proteins were eluted with $300 \mu \mathrm{l}$ of Flag peptide (Sigma), at a concentration of $5 \mu \mathrm{g} / \mu \mathrm{l}$ in Tris-buffered saline (TBS), for 30 minutes at $4{ }^{\circ} \mathrm{C}$. The eluted proteins were collected by transferring the resin mixture to a Pierce Micro-spin column (ThermoScientific) and centrifuging at 10,000 $\times g$ for 3 minutes.

\subsection{Electrophoresis}

Samples were subjected to SDS-PAGE and native PAGE as described $[20,21]$ except 4-15\% non-denaturing polyacrylamide gradient gels, as well as $10 \%, 12 \%$ and $11-15 \%$ step gradient SDS-PAGE gels were used as indicated. For all gels, the migration of molecular size standards is indicated to the left of each gel image in the figures. The $4-15 \%$ gradient gels were precast Mini-PROTEAN TGX (Bio-Rad) while all the others were poured in lab. For native

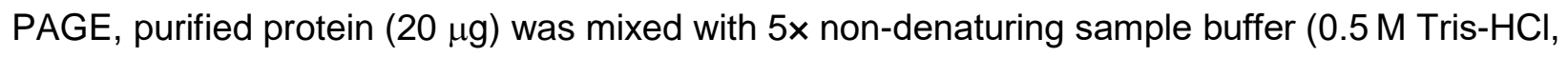
$\mathrm{pH} 8.8,50 \%(\mathrm{v} / \mathrm{v})$ glycerol, traces of bromophenol blue). Non-denaturing gels were run at $60 \mathrm{~V}$ for 10 hours at $4{ }^{\circ} \mathrm{C}$. Substrate overlay assay was carried out as described [20]. Native gels were stained with Imperial Protein Stain (ThermoScientific). Loading control samples were run on reducing 12\% SDS-PAGE. All SDS-PAGE gels were stained with GelCode blue (ThermoScientific).

For Western blotting, samples were run on 10\% SDS-PAGE gels and transferred to PVDF membrane. The membrane was blocked overnight with $5 \%(w / v)$ non-fat milk in TBS then incubated with anti-Flag antibody (Sigma), diluted 1:2000 in 5\% (w/v) non-fat milk in TBS, for 1 hour at room temperature with gentle rocking. The membrane was washed 3 times with TT Buffer $(0.1 \%(v / v)$ Tween-20 in TBS) for 5 minutes and then incubated for 1 hour at room temperature with gentle rocking in TT buffer containing secondary goat anti-mouse $\operatorname{lgG}_{1}$ 
antibody (SouthernBiotech) diluted 1:5000. The membrane was again washed 3 times as above before adding ECL substrate (ThermoFisher) and exposing to film.

\subsection{Disulfide Crosslinking}

Disulfide crosslinking was performed as described [20]. Crosslinked and non-crosslinked samples were mixed with $2 \times$ SDS sample buffer without DTT and loaded on to $11-15 \%$ SDS-

PAGE step gradient gels. Where indicated, a $25 \mu$ aliquot of each sample was reduced with $2 \mu l$ of $1 \mathrm{M}$ DTT at room temperature for 15 minutes.

\subsection{Depletion Assay}

Yeast cell pellets were lysed and CP purified with anti-Flag agarose resin as above. The Flag eluates were subjected to depletion via immobilized-cobalt affinity resin (ICAR) as described [21], with the following modifications. The samples were applied to $150 \mu$ of resin (Talon resin; Clontech) for 1 hour at $4{ }^{\circ} \mathrm{C}$ with gentle rocking. The flow through from the first ICAR depletion was subjected to a second round of ICAR using a fresh $150 \mu$ l of resin.

\subsection{Proteomic Analysis}

Slices cut from indicated gels were submitted to the Indiana University School of Medicine Proteomics Core Facility (IUSM-PCF) to identify proteins by LC-MS/MS. Summarized and annotated data are presented in table format throughout the manuscript and supplementary information. The data analysis files provided by IUSM-PCF in Microsoft Excel format can be found in the accompanying Data In Brief article [22].

\section{Results}

Initially, we set out to study the formation and function of $\alpha 4-\alpha 4$ proteasomes in yeast. We employed a crosslinking strategy [14] using an engineered $\alpha 4$ subunit ( $\alpha 4 C C)$ with a Cterminal Flag epitope (Figure 1A). When $\alpha 4-\alpha 4$ proteasomes are present, the $\alpha 4 \mathrm{CC}$ subunit gives rise to a diagnostic $\alpha 4$ dimer on non-reducing SDS-PAGE under mildly oxidizing 
conditions. This dimer was readily observed when CP was purified from $\alpha 3 \Delta$ yeast (Figure 1B, lane 4). However, the $\alpha 4$ dimer was not observed in the wild-type sample (lane 2) consistent with previous observations that $\alpha 4-\alpha 4$ proteasomes are not detectable (at least using this approach) in wild-type yeast cells [9,14].

Interestingly, additional bands appeared near the top of the SDS-PAGE gel under nonreducing conditions. Like the $\alpha 4$ dimer, these species required the presence of $\alpha 4 \mathrm{CC}$ and disappeared under reducing conditions (Figure 1B); they also failed to form in the absence of added oxidant (Figure 1C). This argues that these slowly migrating species were the result of the engineered disulfide crosslink, just like the $\alpha 4$ dimer. However, unlike the $\alpha 4$ dimer, these species were also present in wild-type cells, albeit at lower levels compared to $\alpha 3 \Delta$ cells (Figure 1B, lanes 2 versus 4). LC-MS/MS analysis confirmed the presence of $\alpha 4$ in these bands (not shown) consistent with the slowly migrating species being a multimer (or multimers) of $\alpha 4$ crosslinked to itself.

To determine if these slowly migrating species represented actual $\alpha 4$-containing high molecular weight complexes (HMWCs), we analyzed Flag-purified CP samples by native PAGE. The major complex in each sample, migrating near the $670 \mathrm{kDa}$ size standard, was the CP (Supplementary Figure 1). Species migrating slower than the CP were likely complexes of CP and BIm10; these were more abundant in the $\alpha 3 \Delta$ samples because CP lacking $\alpha 3$ are constitutively open and BIm10 preferentially binds to CP with an open (or disordered) gate [23]. Species migrating faster than the CP were also observed, some of which were likely CP assembly intermediates (Supplementary Figure 1, bracket); if $\alpha 4$ HMWCs were present, they would likely be found among them. To better visualize these faster migrating species, all of our subsequent native PAGE gels were loaded with an excess of protein (Figure 2A).

We excised several of these faster migrating species and submitted them for analysis by LC-MS/MS (Supplementary Figures 2 to 7, and also [22]). As expected, all six bands analyzed 
suggested the presence of CP assembly intermediates. For example, band 1 contained all $\alpha$ subunits, all $\beta$ subunits except $\beta 7$, as well as the assembly factors Ump1, and Pba1-Pba2 (Figure 2A and Supplementary Figure 2). This is consistent with band 1 containing the $15 \mathrm{~S}$ assembly intermediate of the CP [6] (alternatively known as the "- $\beta 7$ half-mer" [10]). Interestingly, the composition of band 3 argued it too contained the $15 S$ (Supplementary Figure 4). Yet band 3 migrated slightly slower than band 1, and was also more intense.

Band 3 was derived from a wild-type yeast strain containing $\alpha 4 \mathrm{CC}$ whereas band 1 was derived from an isogenic strain containing (non-crosslinkable) $\alpha 4$. Hence, the difference in their electrophoretic mobility can be accounted for in two, not mutually exclusive, ways. On the one hand, to generate $\alpha 4 \mathrm{CC}$, two amino acids with non-ionizable sidechains were replaced by cysteines, which can be appreciably ionized at the $\mathrm{pH}$ of the resolving gel; this could affect the charge of $\alpha 4 \mathrm{CC}$-containing species and hence contribute to a migration difference of $15 \mathrm{~S}$ in band 1 versus band 3 . On the other hand, the increased intensity of band 3 could represent a novel species not found in band 1 i.e. the sought-after $\alpha 4$ HMWCs. Consistent with the latter scenario, we observed an excess of spectral counts for peptides derived from the $\alpha 4$ subunit, compared to those derived from other $\alpha$ subunits, in band 3 (Figure 2B). This vast excess was observed for all bands from samples containing $\alpha 4 \mathrm{CC}$ (bands 3 to 6 ) and argued that these bands contained considerably more $\alpha 4$ than the other subunits. The simplest explanation was that bands 3 through 6 included not only CP assembly intermediates but also comparably sized HMWCs consisting of $\alpha 4 C C$ (Supplementary Figures 2 to 7 ). The subunit composition of bands 1 through 6 , including the excess of $\alpha 4$ in bands 3 to 6 , was reproducible (Supplementary Figure 8).

To confirm the existence of these novel $\alpha 4$ HMWCs as independent species, we pursued a depletion approach (Supplementary Figure 9). Initially, we chose to do this in an $\alpha 3 \Delta$ background because our data suggested these HMWCs are more abundant when $\alpha 3$ is deleted 
(Figures $1 \mathrm{~B}$ and 2). We employed a strain in which $\alpha 2$ was $\mathrm{C}$-terminally tagged with a tandem epitope consisting of a hexahistidine tag (his tag) and a Flag tag ( $\alpha 2 \mathrm{HF})$. We crossed $\alpha 2 \mathrm{HF}$ and $\alpha 4 \mathrm{CC} \alpha 3 \Delta$ yeast to generate the $\alpha 4 \mathrm{CC} \alpha 3 \Delta \alpha 2 \mathrm{HF}$ experimental depletion strain. We prepared lysates from cultures of the experimental depletion strain, and its two parental strains as controls ( $\alpha 4 \mathrm{CC} \alpha 3 \Delta$ and $\alpha 2 \mathrm{HF}$ ), and isolated all Flag-tagged species. We subjected the Flag eluates to both native and SDS-PAGE (Figures 3A and 3B, lanes 1 to 3). The CP was the major species in all the samples and faster migrating species were also observed. The faster migrating species in the two $\alpha 4 \mathrm{CC}$ containing strains (Figure $3 \mathrm{~A}$, lanes 1 and 3 ) were the equivalent of bands 5 and 6 in Figure 2A. As expected, these two bands were absent in the $\alpha 2 \mathrm{HF}$ strain (Figure $3 \mathrm{~A}$, lane 2 ) because this strain did not contain Flag-tagged $\alpha 4$; it is not possible to isolate any $\alpha 4$ HMWCs from this strain. The Flag eluates were depleted of any histagged proteins via two rounds of binding to immobilized cobalt affinity resin (ICAR; Supplementary Figures 9 and 10). Protein complexes not containing his-tagged components would remain in the second "his flow through" (Figures $3 \mathrm{~A}$ and $3 \mathrm{~B}$, lanes 4 to 6).

The his flow through from the $\alpha 4 \mathrm{CC} \alpha 3 \Delta$ parental control looked like its corresponding Flag eluate; since no $\alpha 2 \mathrm{HF}$ was present, none of the species were retained on ICAR (Figures $3 \mathrm{~A}$ and $3 \mathrm{~B}$, lane 4; Supplementary Figure 10, lane 1). The his flow through of the $\alpha 2 \mathrm{HF}$ parental control was nearly completely empty; since the only Flag-tagged protein in this strain was also his-tagged, all Flag-containing species were depleted by ICAR (Figures 3A and 3B, lane 5), though a tiny amount of CP did escape depletion. In the his flow through of the experimental strain, nearly all of the CP was depleted, except for the tiny amount also present in the $\alpha 2 \mathrm{HF}$ sample (Figure 3A, lane 6). Importantly, the two species migrating faster than CP were recovered in considerable amounts (bands 7 and 8), consistent with these species containing Flag-tagged $\alpha 4$ but not his-Flag-tagged $\alpha 2$. LC-MS/MS analysis confirmed the dominant species in each band was $\alpha 4$, though a considerable amount of the closely related Hsp70 
paralogs, Ssa1p and Ssa2p, were present in band 8. The LC-MS/MS results were corroborated by SDS-PAGE showing $\alpha 4$ as the major species and a weaker Hsp70-containing band (Figure $3 B$, lane 6). We repeated the depletion analysis and included the wild-type (i.e. non $\alpha 3 \Delta$ ) yeast strain for comparison, which confirmed that these $\alpha 4$ HMWCs are also present in wild-type cells (Figure 3C).

Finally, we subjected aliquots of the his flow through from the $\alpha 4 \mathrm{CC} \alpha 3 \Delta \alpha 2 \mathrm{HF}$ depletion strain (containing $\alpha 4$ HMWCs) to SDS-PAGE under reducing and non-reducing conditions; prior to electrophoresis, the samples were either treated (or not treated) with $\mathrm{CuCl}_{2}$ to initiate crosslinking (Figure 4A). Under non-reducing conditions, all the $\alpha 4$ migrated as distinct species near the top of the resolving gel. This confirmed that that the $\alpha 4$ HMWCs isolated in the depletion analysis were identical to the slowly migrating $\alpha 4$ species initially observed by nonreducing SDS-PAGE (Figure 1B), and consisted of $\alpha 4$ crosslinked to itself. This was further verified by Western blotting which demonstrated an expected $\alpha 4$ laddering pattern (Figure 4B); this occurs when some of the $\alpha 4$ HMWCs in the sample are not completely crosslinked. It is notable that $\mathrm{CuCl}_{2}$ was not required to observe the $\alpha 4 \mathrm{HMWCs}$ in Figure 4, unlike in Figure $1 \mathrm{~B}$. We have shown before that protracted purification protocols, especially involving ICAR, are sufficiently oxidizing to allow crosslinking to occur even in the absence of exogenous oxidant [21] (see also Supplementary Note).

\section{Discussion}

We provide evidence that the $\alpha 4$ subunit exists in high molecular weight complexes (HMWCs) outside of the CP in vivo (Figures 1B and 3). These non-canonical species are likely ring-shaped because their discovery was enabled by a cross-linking strategy developed to detect adjacent $\alpha 4$ subunits within the same $\alpha$-ring [14] and because we observed a distinctive $\alpha 4$ laddering pattern that would be expected if these were rings (Figure 4B). The seven 
crosslinked bands in the laddering pattern could be evidence for an octameric ring, though it is also possible that two of the bands (presumably the two highest) each contain seven copies of $\alpha 4$. This would occur if one band is derived from a completely crosslinked (i.e. closed) heptameric ring and one band is derived from a heptameric ring with one crosslink missing (i.e. an open chain of 7 subunits). Circular and linear topologies of a protein can migrate differently on SDS-PAGE [24].

Non-canonical rings containing different combinations of $\alpha$ subunits have been observed before, but only when recombinantly produced in bacteria $[16,17,18,19]$. Thus, it was not clear if their recombinant existence was relevant physiologically. Ours is the first evidence that such complexes exist in vivo, in wild-type cells. The two species we observed on native PAGE (Figure 3A, bands 7 and 8 ) could be single and double $\alpha 4$ rings that exist in equilibrium. There are several reasons why these particular $\alpha 4$ HMWCs escaped detection until now, even though the $\alpha 4-\alpha 4$ crosslinking approach was used before $[9,14]$. First, the previous studies were interested in detecting $\alpha 4-\alpha 4$ proteasomes, specifically the characteristic $\alpha 4-\alpha 4$ dimers. Their analysis was not optimized to detect HMWCs, which likely failed to enter the resolving gel. Here, using a lower percentage step gradient gel, we were able to detect these HMWCs (Figure 1B) which just barely entered the resolving gel. Second, the HMWCs may not be as stable as $\mathrm{CP}$, especially when analyzed by native PAGE; the presence of crosslinkable $\alpha 4$ was needed to clearly visualize them (Figure 3 and Supplementary Figure 8; see also Supplementary Note). Finally, these HMWCs are comparable in size to known CP intermediates (such as 13S, 15S, and half-proteasome species) and can thus be obscured by them. The excess of spectral counts for $\alpha 4$-derived peptides was consistent with this (Figure 2B) and our depletion experiments (Figure 3) confirmed it. We suggest increased vigilance in interpreting native PAGE data for proteasome assembly. 
The purpose of these $\alpha 4$ HMWCs remains to be determined. Recently, $\alpha 4-G F P$ was found to localize into puncta in the absence of the Pba3-Pba4 chaperone [12]. It is not clear if these "aggregates" of $\alpha 4$ are related to the $\alpha 4$ HMWCs here, but we note that the HMWCs are already present in wild-type yeast cells. The observation that levels of HMWCs can increase, such as when $\alpha 3$ is deleted, suggests their levels could be regulated. This regulation might involve Ssa1/Ssa2 proteins, which associate tightly with HMWCs, surviving the extended depletion protocol.

Our data are consistent HMWCs having a physiological role, though we cannot yet rule out the possibility that these are misassembly products destined for removal by the protein quality control machinery. However, the recent study which identified $\alpha 4-\alpha 4$ proteasomes in mammalian cells, using the same crosslinking approach as here, also acknowledged higher molecular weight species containing $\alpha 4$ in vivo [15]. Though these species were not studied further, we note with interest their observation of an $\alpha 4$ laddering pattern (for example, Figure $2 \mathrm{E}$ in [15]) similar to what we observed here (Figure 4B). This raises the possibility that the formation of $\alpha 4 \mathrm{HMWCs}$, like the formation of $\alpha 4-\alpha 4$ proteasomes, is evolutionarily conserved.

\section{Acknowledgments}

The authors thank Dilrajkaur Panfair for comments on this manuscript and Mark Hochstrasser (Yale University) for providing yeast strains. This work was supported by a Research Support Funds Grant from Indiana University-Purdue University Indianapolis to A.R.K. 


\section{References}

[1] F. Beck, P. Unverdorben, S. Bohn, A. Schweitzer, G. Pfeifer, E. Sakata, S. Nickell, J.M. Plitzko, E. Villa, W. Baumeister, F. Forster, Near-atomic resolution structural model of the yeast 26S proteasome, Proc Natl Acad Sci U S A 109 (2012) 14870-14875.

[2] P.C. da Fonseca, J. He, E.P. Morris, Molecular Model of the Human 26S Proteasome, Mol Cell 46 (2012) 54-66.

[3] M. Groll, L. Ditzel, J. Lowe, D. Stock, M. Bochtler, H.D. Bartunik, R. Huber, Structure of 205 proteasome from yeast at 2.4 A resolution, Nature 386 (1997) 463-471.

[4] G. Hu, G. Lin, M. Wang, L. Dick, R.M. Xu, C. Nathan, H. Li, Structure of the Mycobacterium tuberculosis proteasome and mechanism of inhibition by a peptidyl boronate, Mol Microbiol 59 (2006) 1417-1428.

[5] J. Lowe, D. Stock, B. Jap, P. Zwickl, W. Baumeister, R. Huber, Crystal structure of the 20 S proteasome from the archaeon T. acidophilum at 3.4 A resolution, Science 268 (1995) 533-539.

[6] M.J. Kunjappu, M. Hochstrasser, Assembly of the 20 S proteasome, Biochim Biophys Acta 1843 (2014) 2-12.

[7] Y. Hirano, T. Kaneko, K. Okamoto, M. Bai, H. Yashiroda, K. Furuyama, K. Kato, K. Tanaka, S. Murata, Dissecting beta-ring assembly pathway of the mammalian $20 \mathrm{~S}$ proteasome, EMBO J 27 (2008) 2204-2213.

[8] P. Chen, M. Hochstrasser, Autocatalytic subunit processing couples active site formation in the 20 s proteasome to completion of assembly, Cell 86 (1996) 961-972.

[9] A.R. Kusmierczyk, M.J. Kunjappu, M. Funakoshi, M. Hochstrasser, A multimeric assembly factor controls the formation of alternative 20S proteasomes, Nat Struct Mol Biol 15 (2008) 237-244. 
[10] X. Li, A.R. Kusmierczyk, P. Wong, A. Emili, M. Hochstrasser, beta-Subunit appendages promote 20 S proteasome assembly by overcoming an Ump1-dependent checkpoint, EMBO J 26 (2007) 2339-2349.

[11] Y. Hirano, K.B. Hendil, H. Yashiroda, S. Iemura, R. Nagane, Y. Hioki, T. Natsume, K. Tanaka, S. Murata, A heterodimeric complex that promotes the assembly of mammalian 20 s proteasomes, Nature 437 (2005) 1381-1385.

[12] K. Takagi, Y. Saeki, H. Yashiroda, H. Yagi, A. Kaiho, S. Murata, T. Yamane, K. Tanaka, T. Mizushima, K. Kato, Pba3-Pba4 heterodimer acts as a molecular matchmaker in proteasome alpha-ring formation, Biochem Biophys Res Commun 450 (2014) 11101114.

[13] P.S. Wani, M.A. Rowland, A. Ondracek, E.J. Deeds, J. Roelofs, Maturation of the proteasome core particle induces an affinity switch that controls regulatory particle association, Nat Commun 6 (2015) 6384.

[14] I. Velichutina, P.L. Connerly, C.S. Arendt, X. Li, M. Hochstrasser, Plasticity in eucaryotic $20 \mathrm{~S}$ proteasome ring assembly revealed by a subunit deletion in yeast, EMBO J 23 (2004) 500-510.

[15] A. Padmanabhan, S.A. Vuong, M. Hochstrasser, Assembly of an Evolutionarily Conserved Alternative Proteasome Isoform in Human Cells, Cell Rep 14 (2016) 2962-2974.

[16] W.L. Gerards, W.W. de Jong, H. Bloemendal, W. Boelens, The human proteasomal subunit HsC8 induces ring formation of other alpha-type subunits, J Mol Biol 275 (1998) 113121.

[17] W.L. Gerards, J. Enzlin, M. Haner, I.L. Hendriks, U. Aebi, H. Bloemendal, W. Boelens, The human alpha-type proteasomal subunit HsC8 forms a double ringlike structure, but does not assemble into proteasome-like particles with the beta-type subunits HsDelta or HsBPROS26, J Biol Chem 272 (1997) 10080-10086. 
[18] K. Ishii, M. Noda, H. Yagi, R. Thammaporn, S. Seetaha, T. Satoh, K. Kato, S. Uchiyama, Disassembly of the self-assembled, double-ring structure of proteasome alpha7 homotetradecamer by alpha6, Sci Rep 5 (2015) 18167.

[19] Y. Yao, C.R. Toth, L. Huang, M.L. Wong, P. Dias, A.L. Burlingame, P. Coffino, C.C. Wang, alpha5 subunit in Trypanosoma brucei proteasome can self-assemble to form a cylinder of four stacked heptamer rings, Biochem J 344 Pt 2 (1999) 349-358.

[20] A.R. Kusmierczyk, M.J. Kunjappu, R.Y. Kim, M. Hochstrasser, A conserved 20S proteasome assembly factor requires a C-terminal $\mathrm{HbYX}$ motif for proteasomal precursor binding, Nat Struct Mol Biol 18 (2011) 622-629.

[21] D. Panfair, A. Ramamurthy, A.R. Kusmierczyk, Alpha-ring Independent Assembly of the 20S Proteasome, Sci Rep 5 (2015) 13130.

[22] L.J. Hammack, A.R. Kusmierczyk, Data on the identity of non-canonical complexes formed from proteasome subunits in vivo., Data in Brief (2016). Submitted.

[23] A. Lehmann, K. Jechow, C. Enenkel, BIm10 binds to pre-activated proteasome core particles with open gate conformation, EMBO Rep 9 (2008) 1237-1243.

[24] H. Iwai, A. Pluckthun, Circular beta-lactamase: stability enhancement by cyclizing the backbone, FEBS Lett 459 (1999) 166-172. 


\section{Figure Legends}

Fig. 1. Detection of novel $\alpha 4$-containing species. (A) Schematic of crosslinkable $\alpha 4$ subunit ( $\alpha 4 C C$ ) and the resulting crosslink from adjacent $\alpha 4$ 's within an $\alpha$-ring. (B) Flag-purified CP from the indicated yeast strains was treated with $\mathrm{CuCl}_{2}$ to initiate crosslinking. Samples were subjected to SDS-PAGE under non-reducing (lanes 1 to 4), or reducing (lanes 5 to 8) conditions. White arrowhead denotes position of $\alpha 4-\alpha 4$ dimer. Black arrowheads denote slowmigrating $\alpha 4$ species. Asterisk denotes migration of the $\alpha 3$ subunit, or its absence, in $\alpha 3 \Delta$ strains. Bracket denotes subunits of the CP. (C) Analysis as in (B) except the samples were not crosslinked.

Fig. 2. Analysis of putative $\alpha 4-c o n t a i n i n g ~ H M W C s$ by native PAGE. (A) Native PAGE of Flag-purified CP from the indicated yeast strains (top panel). Arrowheads denote migration of CP and of CP assembly intermediates (1 to 6). No protein was loaded in lanes labeled "blank" to minimize effect of spillover between samples. To verify equal loading of proteins, aliquots of the Flag-purified material were subjected to SDS-PAGE (bottom panel). (B) Contents of bands 1 to 6 in (A) were analyzed by LC-MS/MS. Graphs indicate the total count of PSMs (peptide spectral matches) for peptides derived from individual $\alpha$ subunits. Numbers below each subunit represent the actual number of peptides identified for that subunit.

Fig. 3. Depletion analysis confirms novel $\alpha 4$ complexes. (A) Aliquots of Flag-purified CP from the indicated yeast strains were subjected to native PAGE (lanes 1 to 3 ). The remainder of the Flag-purified material was depleted of his-tagged proteins via two rounds of binding to immobilized-cobalt affinity resin (ICAR). Aliquots of the flow through from the second binding to ICAR were loaded on the same native PAGE gel (lanes 4 to 6). Arrowheads denote the position of $\mathrm{CP}$ and two faster migrating species (7 and 8) remaining following depletion. Contents of bands 7 and 8 are shown at right. (B) Aliquots of the samples in (A) were also analyzed by 
SDS-PAGE. (C) Depletion analysis was repeated to demonstrate that wild-type yeast strains also possess the novel $\alpha 4$ complexes.

Fig. 4. Quaternary structure of $\alpha 4$ HMWCs. (A) Aliquots of the $\alpha 4 \mathrm{HMWCs}$ isolated by depletion analysis, as in Figure 3, were incubated in the presence or absence of $\mathrm{CuCl}_{2}$ to induce crosslinking. Samples were subjected to SDS-PAGE under non-reducing and reducing conditions. (B) Increasing amounts of $\alpha 4$ HMWCs were subjected to SDS-PAGE under nonreducing conditions $(2,4$, and $8 \mu \mathrm{l})$, or reducing conditions $(8 \mu \mathrm{l}+\mathrm{DTT})$, followed by Western blotting with anti-Flag antibody. Arrowheads denote position of crosslinked products containing increasing copies of $\alpha 4$. The position of the monomer is denoted by $\alpha 4 \mathrm{CC}$. 
A

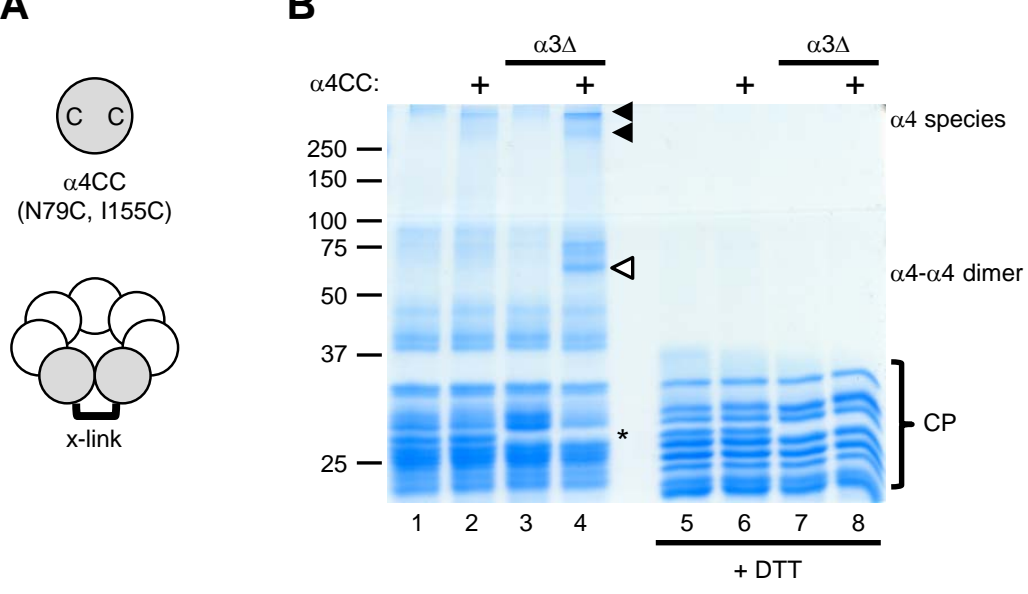

C

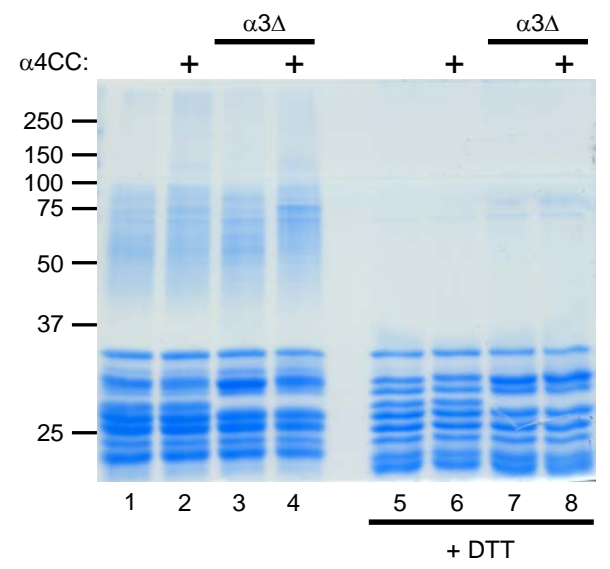




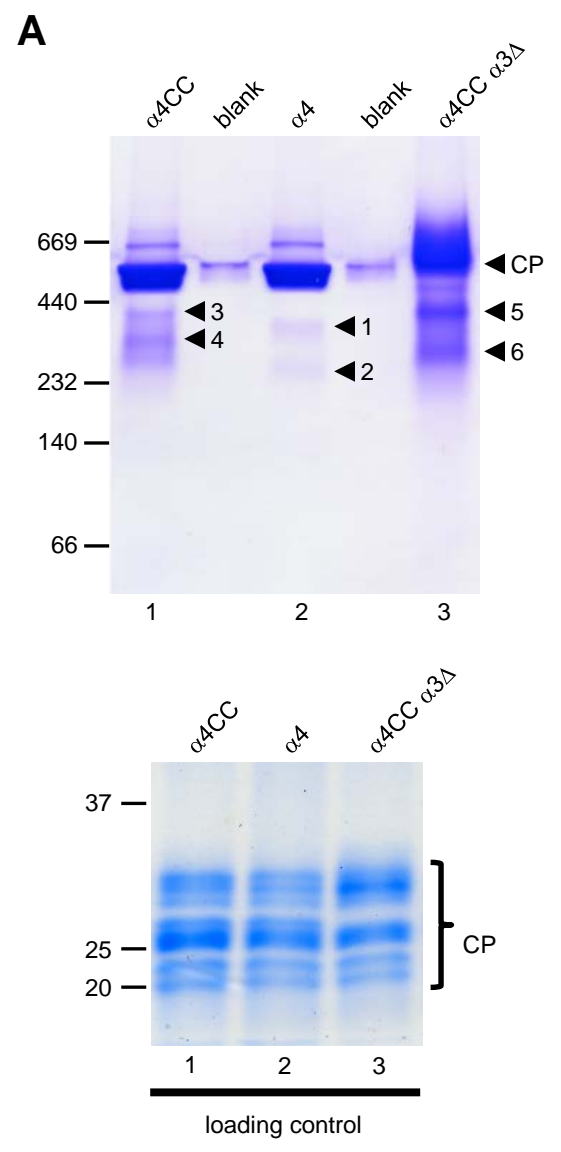

B
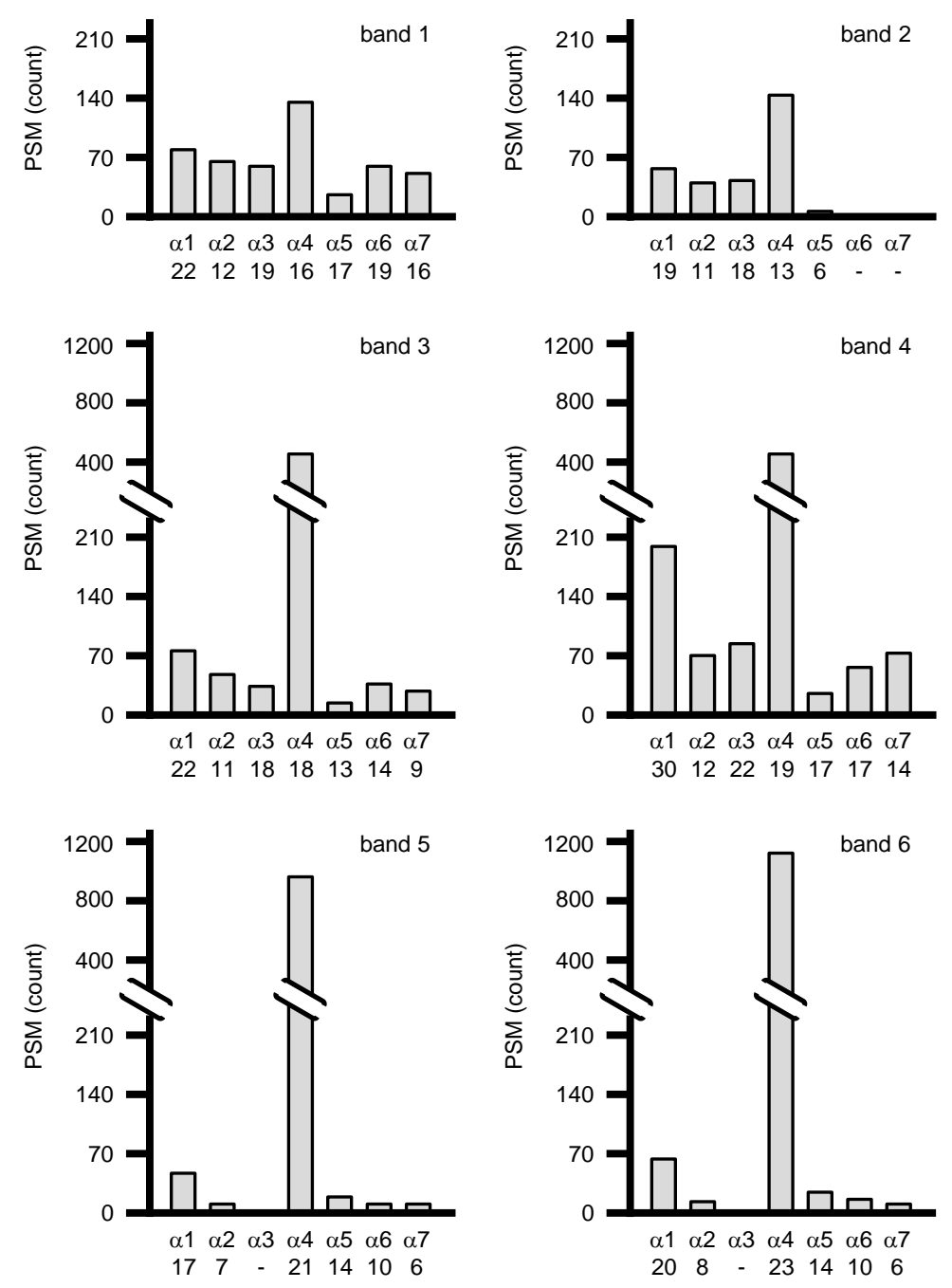


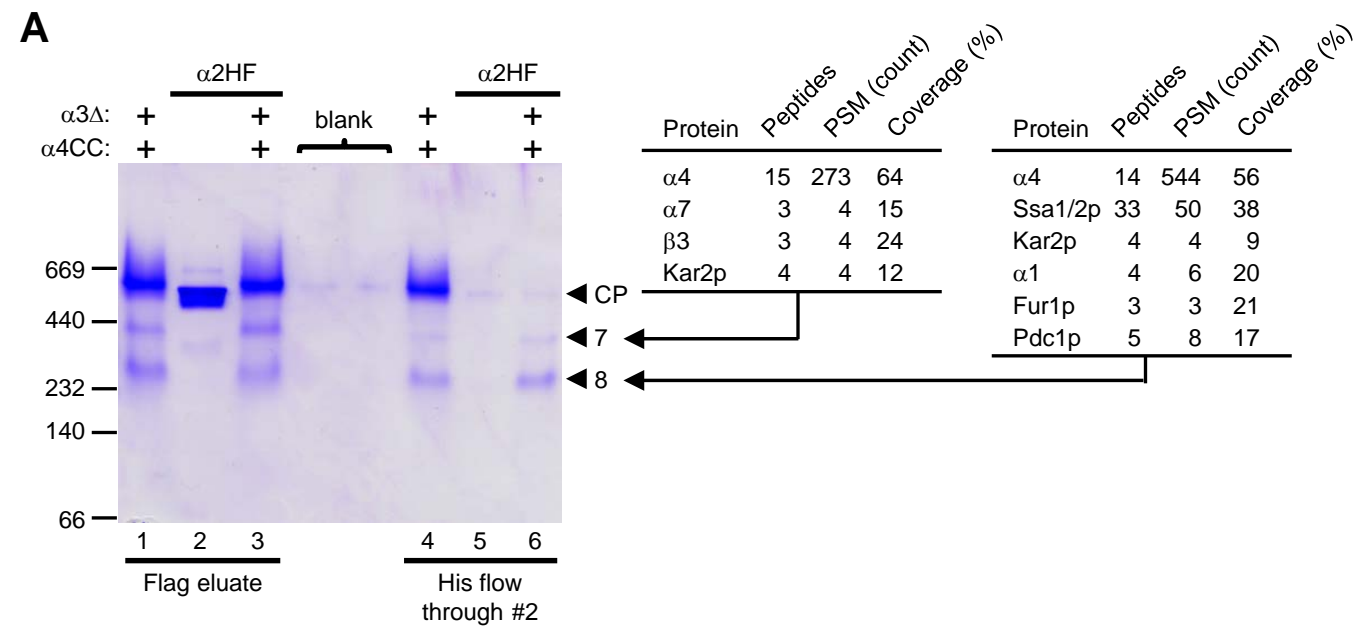

B

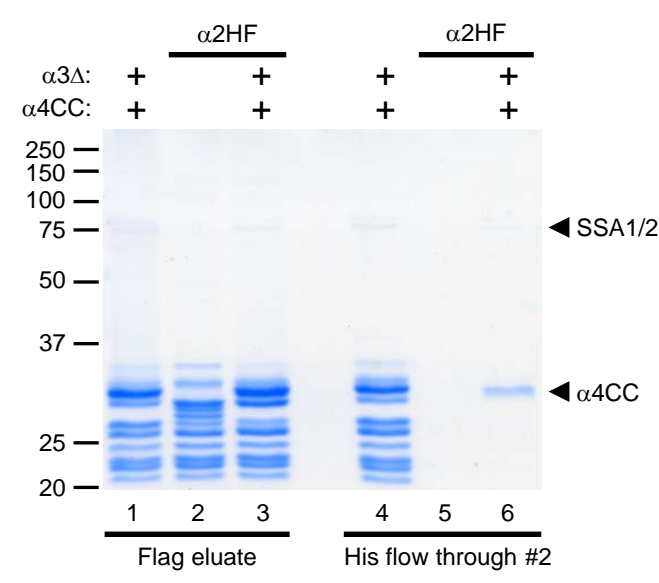

C

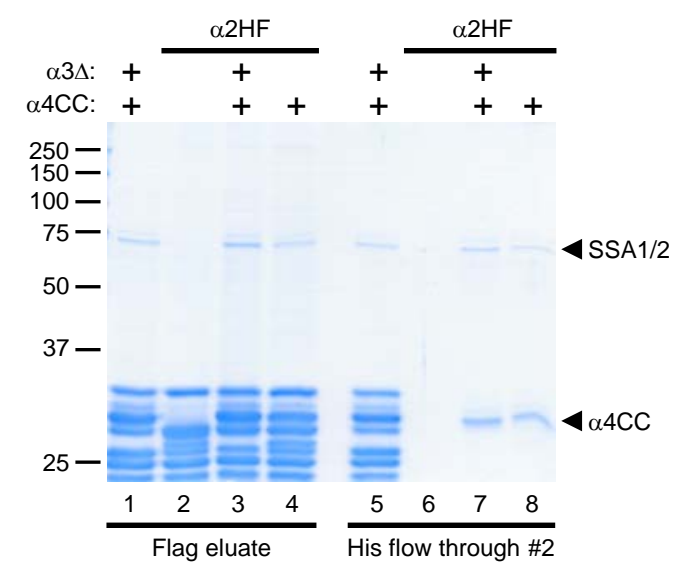


A

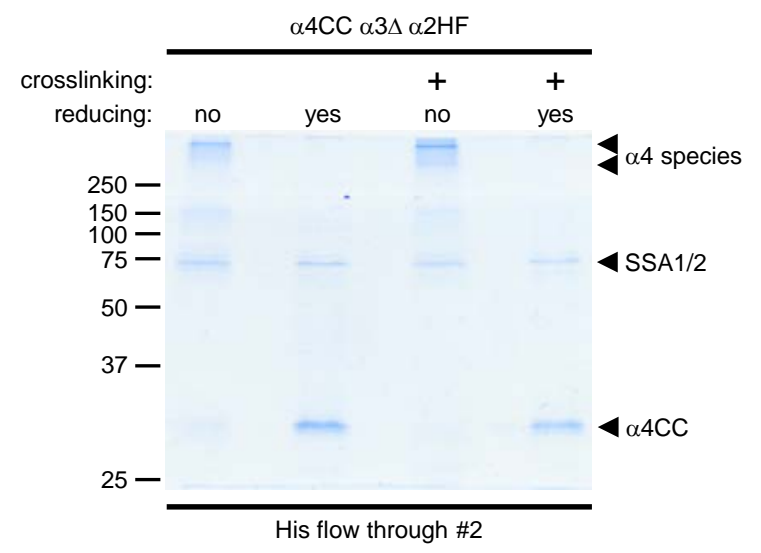

B

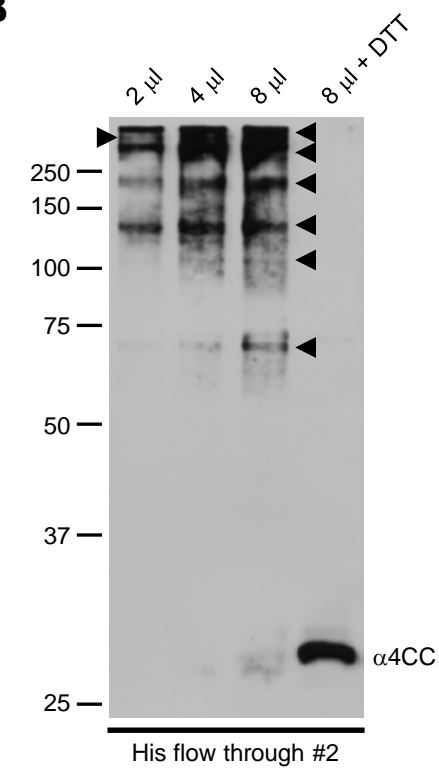


Supplementary Information for

"Assembly of proteasome subunits into non-canonical complexes in vivo" Lindsay J. Hammack and Andrew R. Kusmierczyk

Supplementary Table 1

Supplementary Figures 1 to 10

Supplementary Note 
Supplementary Table 1: Yeast Strains used in this study Name Genotype

AKY889 MATa his3- 200 leu2-3,112 ura3-52 lys2-801 trp1-1 pre6 $:$ :HIS3 [pRS315 pre6-flag]

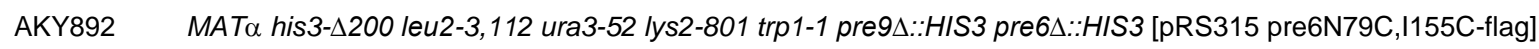

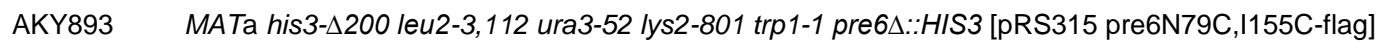

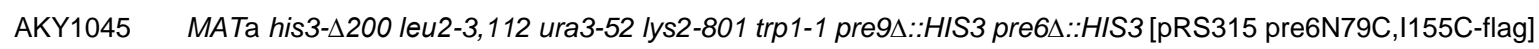

AKY1066 MATa his3- M200 leu2-3,112 ura3-52 lys2-801 trp1-1 pre8-HF(URA3)

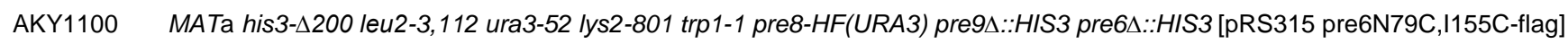

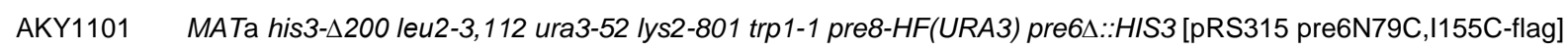

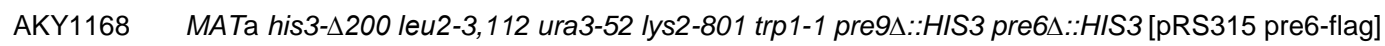

All strains generated in this study. 

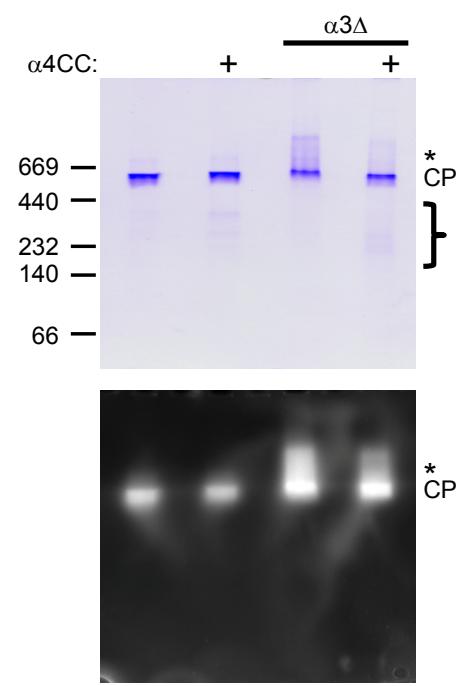

\section{Supplementary Figure 1. Flag-purified CP.}

Yeast lysates from the indicated strains were bound to Flag resin. The Flag-purified CP $(4 \mu \mathrm{g})$ was subjected to native PAGE and substrate overlay assay using the fluorogenic peptide substrate Suc-LLVY-AMC. The gel was stained with GelCode blue and the migration of molecular size standards is indicated at left. CP denotes migration of the core particle. Asterisk denotes migration of CP-BIm10 complexes. Faster migrating species, likely containing $\mathrm{CP}$ assembly intermediates, are denoted by the bracket. This figure demonstrates the requirement for loading of excess protein onto native PAGE in order to better visualize the faster migrating species of interest. Consequently, all subsequent native PAGE were loaded with $20 \mu \mathrm{g}$ protein. 


\begin{tabular}{|c|c|c|c|c|c|c|c|c|}
\hline$\alpha 1$ & 22 & 78 & 88 & Pba1 & 12 & 54 & 62 & \\
\hline$\alpha 2$ & 12 & 65 & 52 & Pba2 & 9 & 27 & 32 & \\
\hline$\alpha 3$ & 19 & 58 & 74 & Ump1 & 3 & 22 & 23 & \\
\hline$\alpha 4$ & 16 & 135 & 79 & Ecm29 & 5 & 5 & 4 & (31) \\
\hline$\alpha 5$ & 17 & 34 & 60 & Rkr1 & 5 & 5 & 10 & \\
\hline$\alpha 6$ & 19 & 60 & 84 & BIm10 & 6 & 8 & 5 & $15 S$ intermediate \\
\hline$\alpha 7$ & 16 & 49 & 61 & Hsp60 & 3 & 4 & 7 & \\
\hline$\beta 1$ & 6 & 7 & 35 & Pfk26 & 9 & 9 & 14 & \\
\hline$\beta 2$ & 9 & 69 & 32 & Tdh2 & 3 & 4 & 21 & \\
\hline$\beta 3$ & 8 & 38 & 47 & & & & & \\
\hline$\beta 4$ & 10 & 20 & 47 & & & & & \\
\hline$\beta 5$ & 8 & 9 & 33 & & & & & \\
\hline$\beta 6$ & 5 & 12 & 35 & & & & & \\
\hline
\end{tabular}

Supplementary Figure 2. Composition of Band 1 from Figure 2.

Results of LC-MS/MS analysis (left) and the identity of the likely CP intermediate and/or CP-subunit-derived species present (right).

\begin{tabular}{|c|c|c|c|c|c|c|c|c|}
\hline$\alpha 1$ & 19 & 57 & 83 & Ump1 & 2 & 8 & 16 & \\
\hline$\alpha 2$ & 11 & 40 & 37 & Rpn3 & 5 & 6 & 15 & \\
\hline$\alpha 3$ & 18 & 43 & 77 & Rpn8 & 4 & 7 & 17 & $\therefore<Y_{22}$ \\
\hline$\alpha 4$ & 13 & 143 & 78 & Rpn9 & 3 & 4 & 14 & $(\alpha 1)$ \\
\hline$\alpha 5$ & 6 & 6 & 29 & BIm10 & 11 & 13 & 6 & ( $\beta 1)^{2}$ \\
\hline$\beta 1$ & 6 & 8 & 36 & Ssc1 & 6 & 8 & 13 & 13S-like intermediate \\
\hline$\beta 2$ & 7 & 41 & 31 & Erg6 & 6 & 8 & 24 & 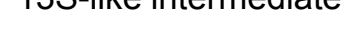 \\
\hline$\beta 3$ & 8 & 27 & 47 & Acs2 & 4 & 8 & 9 & \\
\hline \multirow[t]{6}{*}{$\beta 4$} & 7 & 15 & 46 & Eno1/2 & 6 & 7 & 21 & \\
\hline & & & & Alo1 & 5 & 5 & 11 & \\
\hline & & & & Adh1 & 4 & 5 & 16 & \\
\hline & & & & Pdc1 & 6 & 11 & 21 & \\
\hline & & & & Pfk26 & 12 & 13 & 19 & \\
\hline & & & & Eft2 & 4 & 5 & 8 & \\
\hline
\end{tabular}

Supplementary Figure 3. Composition of Band 2 from Figure 2.

Results of LC-MS/MS analysis (left) and the identity of the likely CP intermediate and/or CP-subunit-derived species present (right). 


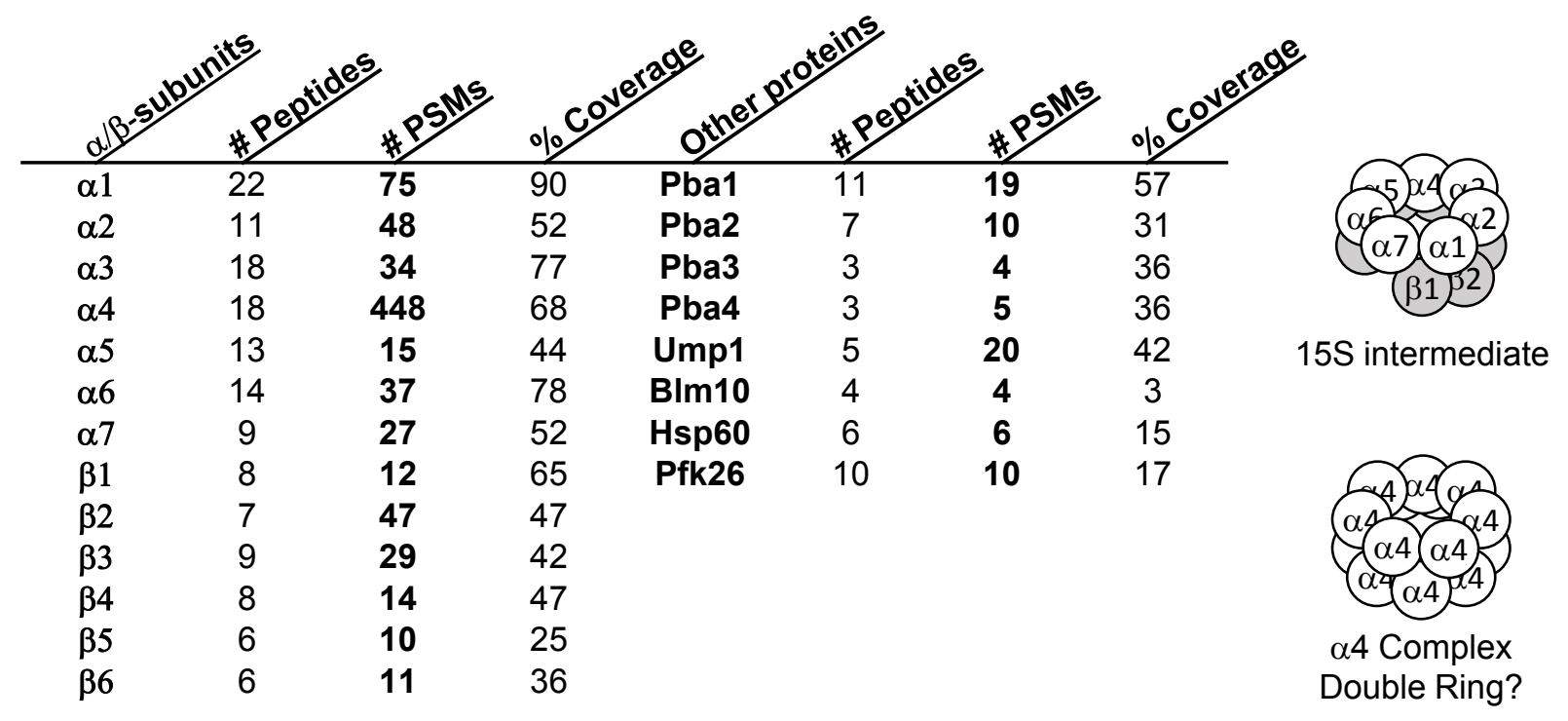

Supplementary Figure 4. Composition of Band 3 from Figure 2.

Results of LC-MS/MS analysis (left) and the identity of the likely CP intermediate and/or CP-subunit-derived species present (right).

\begin{tabular}{|c|c|c|c|c|c|c|c|c|}
\hline$\alpha 1$ & 30 & 198 & 93 & Pba1 & 11 & 28 & 57 & \\
\hline$\alpha 2$ & 12 & 70 & 52 & Pba2 & 6 & 13 & 28 & (2) \\
\hline$\alpha 3$ & 22 & 84 & 91 & Pba4 & 5 & 10 & 37 & $32)$ \\
\hline$\alpha 4$ & 19 & 506 & 67 & Ump1 & 5 & 51 & 46 & \\
\hline$\alpha 5$ & 17 & 25 & 62 & BIm10 & 13 & 17 & 8 & 15S-like intermediate \\
\hline$\alpha 6$ & 17 & 57 & 83 & Rkr1 & 6 & 8 & 5 & \\
\hline$\alpha 7$ & 14 & 72 & 63 & Ssa1 & 10 & 12 & 24 & \\
\hline$\beta 2$ & 13 & 85 & 59 & Ssa2 & 11 & 13 & 28 & \\
\hline$\beta 3$ & 8 & 49 & 43 & Kar2 & 4 & 4 & 8 & \\
\hline$\beta 4$ & 9 & 48 & 44 & Tdh3 & 6 & 11 & 34 & \\
\hline$\beta 5$ & 5 & 5 & 25 & Pfk26 & 10 & 10 & 17 & \\
\hline$\beta 6$ & 2 & 6 & 18 & & & & & Single Ring? \\
\hline
\end{tabular}

Supplementary Figure 5. Composition of Band 4 from Figure 2.

Results of LC-MS/MS analysis (left) and the identity of the likely CP intermediate and/or CP-subunit-derived species present (right). 


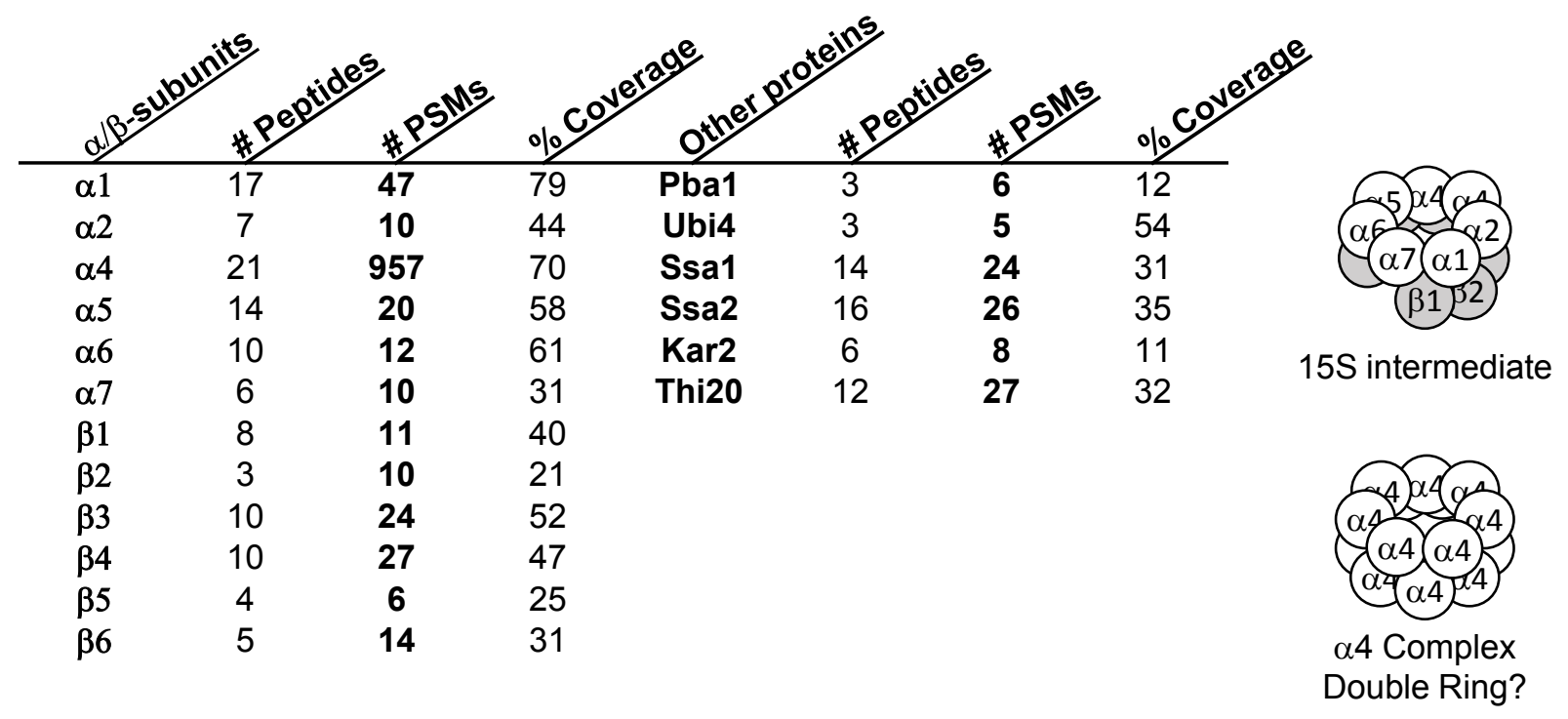

Supplementary Figure 6. Composition of Band 5 from Figure 2.

Results of LC-MS/MS analysis (left) and the identity of the likely CP intermediate and/or CP-subunit-derived species present (right).

\begin{tabular}{|c|c|c|c|c|c|c|c|c|}
\hline$\alpha 1$ & 20 & 63 & 79 & Pba1 & 8 & 19 & 46 & \\
\hline$\alpha 2$ & 8 & 13 & 44 & Pba3 & 4 & 5 & 45 & \\
\hline$\alpha 4$ & 23 & 1120 & 74 & Ubi4 & 3 & 4 & 54 & (31) \\
\hline$\alpha 5$ & 14 & 26 & 57 & Ssa1 & 34 & 61 & 59 & \\
\hline$\alpha 6$ & 10 & 16 & 63 & Ssa2 & 32 & 58 & 55 & $15 \mathrm{~S}$ intermediate \\
\hline$\alpha 7$ & 6 & 10 & 31 & Kar2 & 15 & 22 & 30 & \\
\hline$\beta 1$ & 8 & 10 & 59 & Thi20 & 21 & 30 & 51 & \\
\hline$\beta 2$ & 7 & 13 & 37 & Eno1/2 & 5 & 6 & 16 & \\
\hline$\beta 3$ & 8 & 31 & 47 & Pdc1 & 2 & 3 & 9 & (a4) \\
\hline$\beta 4$ & 9 & 49 & 47 & Tdh3 & 3 & 6 & 17 & \\
\hline$\beta 5$ & 5 & 6 & 25 & & & & & $\alpha 4$ Complex \\
\hline$\beta 6$ & 6 & 18 & 40 & & & & & Single Ring? \\
\hline
\end{tabular}

Supplementary Figure 7. Composition of Band 6 from Figure 2.

Results of LC-MS/MS analysis (left) and the identity of the likely CP intermediate and/or CP-subunit-derived species present (right). 


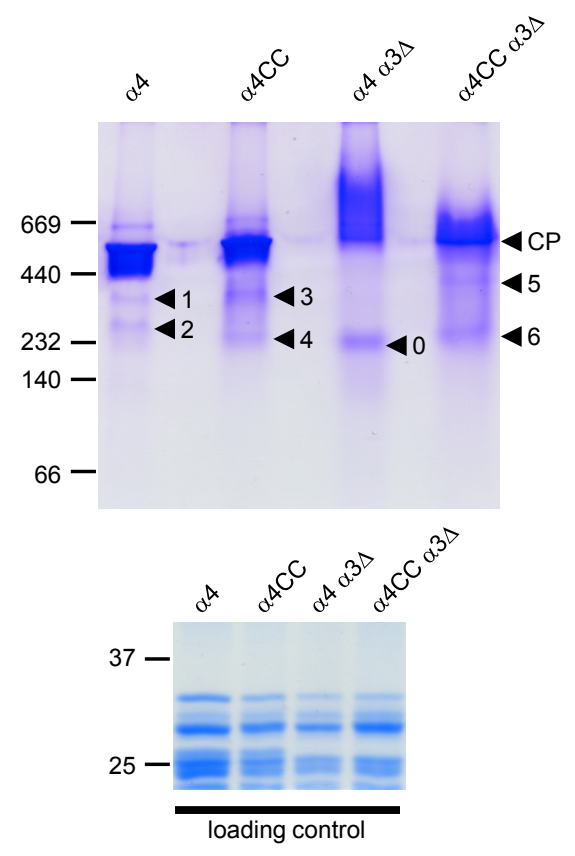

\begin{tabular}{|c|c|c|c|c|c|c|c|c|c|c|c|c|c|c|}
\hline & \multicolumn{2}{|c|}{ band1 } & \multicolumn{2}{|c|}{ band 2} & \multicolumn{2}{|c|}{ band 3} & \multicolumn{2}{|c|}{ band 4} & \multicolumn{2}{|c|}{ band 5} & \multicolumn{2}{|c|}{ band 6} & \multicolumn{2}{|c|}{ band 0} \\
\hline & peptides & PSM & peptides & PSM & peptides & PSM & peptides & PSM & peptides & PSM & peptides & PSM & peptides & PSM \\
\hline$\alpha 1$ & 23 & 123 & 23 & 134 & 24 & 186 & 12 & 37 & 21 & 76 & 22 & 113 & 12 & 40 \\
\hline$\alpha 2$ & 11 & 46 & 10 & 49 & 11 & 70 & 8 & 13 & 9 & 22 & 14 & 47 & 10 & 20 \\
\hline$\alpha 3$ & 19 & 64 & 17 & 64 & 20 & 83 & 16 & 41 & $3^{*}$ & $4^{*}$ & - & - & - & - \\
\hline$\alpha 4$ & 18 & 268 & 19 & 160 & 16 & 760 & 16 & 604 & 16 & 787 & 16 & 867 & 16 & 446 \\
\hline$\alpha 5$ & 17 & 49 & 7 & 9 & 17 & 36 & 9 & 12 & 14 & 26 & 17 & 39 & 14 & 19 \\
\hline$\alpha 6$ & 17 & 55 & - & - & 16 & 57 & 5 & 6 & 13 & 31 & 10 & 15 & 4 & 5 \\
\hline$\alpha 7$ & 12 & 45 & 3 & 5 & 13 & 89 & 3 & 3 & 8 & 29 & 7 & 13 & - & - \\
\hline$\beta 1$ & 10 & 17 & - & - & 4 & 5 & - & - & 12 & 22 & 14 & 26 & 8 & 10 \\
\hline$\beta 2$ & 11 & 67 & 10 & 53 & 12 & 124 & 6 & 13 & 8 & 31 & 10 & 61 & 5 & 13 \\
\hline$\beta 3$ & 8 & 41 & 9 & 46 & 10 & 50 & 6 & 9 & 9 & 39 & 12 & 71 & 12 & 79 \\
\hline$\beta 4$ & 8 & 24 & 9 & 30 & 9 & 39 & 7 & 17 & 8 & 24 & 14 & 106 & 11 & 104 \\
\hline$\beta 5$ & 9 & 23 & 3 & 4 & 7 & 10 & - & - & 6 & 17 & 9 & 23 & 4 & 4 \\
\hline$\beta 6$ & 7 & 26 & 3 & 9 & 5 & 17 & 3 & 13 & 10 & 46 & 12 & 54 & 4 & 19 \\
\hline$\beta 7$ & 7 & 51 & - & - & 5 & 21 & 4 & 7 & 8 & 67 & 6 & 41 & 3 & 9 \\
\hline
\end{tabular}

\section{Supplementary Figure 8. Analysis of putative $\alpha 4$-containing HMWCs by native PAGE.}

This is essentially a repeat of the experiment described in Figure 2, except one additional sample ( $\alpha 4$ $\alpha 3 \Delta$ ) was included. Flag-purified CP from the indicated yeast strains was subjected to native PAGE on 4$15 \%$ gels followed by staining with Imperial Stain (top panel). To verify equal loading of proteins, aliquots of the material analyzed by native PAGE were withdrawn and subjected to SDS-PAGE on $12 \%$ gels, followed by staining with GelCode blue (middle panel). The position of molecular size standards for each gel is indicated on the left. Arrowheads denote migration of CP and of CP assembly intermediates. Bands 1 to 6 on this gel are equivalent to bands 1 to 6 on the native gel in Figure 2 . The contents of the indicated bands was analyzed by LC-MS/MS following their excision from the gel, and the $\alpha$ and $\beta$ subunit contents of the bands is shown (bottom panel). Red denotes the excess in PSMs for the $\alpha 4$ subunit, as observed in Figure 2. Bands 5, 6, and 0 are derived from $\alpha 3 \Delta$ strains, and while bands 6 and 0 do not exhibit peptides derived from $\alpha 3$ (as expected), there are a few $\alpha 3$ peptides in band 5 (asterisk). However, the low number of PSMs for $\alpha 3$, compared to the other CP subunits in this sample, argues that this is likely an experimental artifact. 


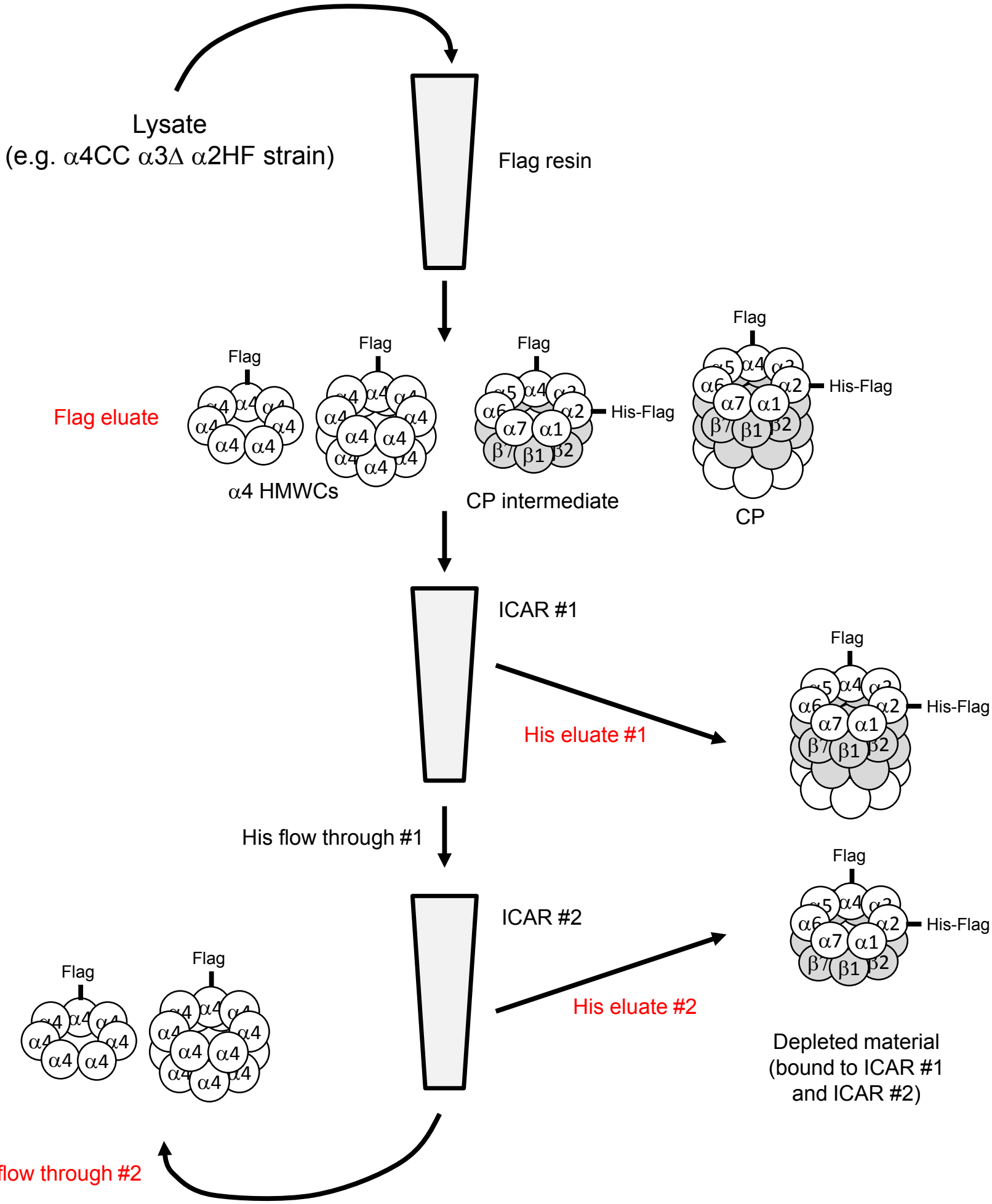

His flow through \#2

\section{Supplementary Figure 9. Depletion strategy.}

Flag tag on subunits $\alpha 4$ and $\alpha 2$ enables the isolation of all CP and CP intermediates, as well as $\alpha 4$ HMWCs. Subjecting the Flag eluates to two rounds of ICAR binding depletes $\alpha 2$-containing species (CP and CP intermediates) because $\alpha 2$ also contains a His tag. For simplicity, the tags are shown on only one subunit in each complex even though all subunits (i.e. $\alpha 2$ and/or $\alpha 4$ ) have this tag. Red denotes samples loaded on various gels shown in this study. 


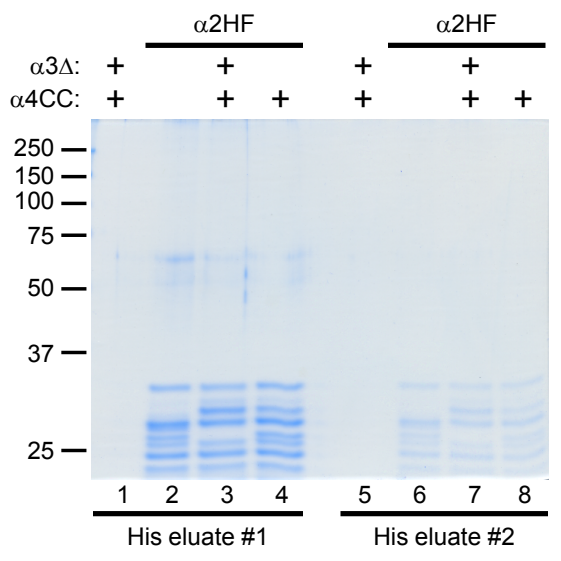

Supplementary Figure 10. Depletion requires more than one round of ICAR binding.

The Flag eluates from Figure $3 \mathrm{C}$ were subjected to depletion of his-tagged proteins by binding to ICAR. The bound material was eluted and subjected to SDS-PAGE (lanes 1 to 4 ). The unbound material (i.e. flow-through \#1) was bound a second time to ICAR using fresh resin. This twicedepleted material was eluted and subjected to SDS-PAGE (lanes 5 to 8). The gel was stained with GelCode blue and the migration of molecular size standards is indicated at left. 


\section{Supplementary Note}

In the Discussion section of the main-text, we argue that $\alpha 4$ HMWCs may not be as stable on native PAGE as other CP-related species, requiring the presence of $\alpha 4 C \mathrm{C}$ to visualize them (Figure 3 and Supplementary Figure 8). It deserves mention that although $\mathrm{CuCl}_{2}$-induced crosslinking was used to observe the $\alpha 4 \mathrm{HMWCs}$ by non-reducing SDS-PAGE in Figure 1B, it was not used prior to analysis of the same samples by native PAGE (Figure 2A). The primary reason for this is that following $\mathrm{CuCl}_{2}$ treatment, the species migrating faster than the $\mathrm{CP}$ appear as a uniform smear on native PAGE (not shown). This treatment is too oxidizing for the multiprotein species to resolve into distinct bands on the native gel and would have resulted in us not being able to identify the $\alpha 4$ HMWCs.

However, we have shown before that experimental conditions during lysis, especially protracted periods of purification, are sufficiently oxidizing to enable (some) crosslinking to occur [1]. This is clearly seen in Figure 4A, whereby the his flow through samples following the lengthy depletion analysis exhibited essentially complete crosslinking of $\alpha 4 \mathrm{CC}$, even in the absence of $\mathrm{CuCl}_{2}$. Contrast this with Figure 1B, which shows the sample after a much shorter protocol (Flag purification only, no depletion) and requires $\mathrm{CuCl}_{2}$ for visualization of the slow migrating $\alpha 4$ species. Finally, the process of electrophoresis is itself oxidizing to proteins ([2] and references therein), and the native PAGE here is carried out over a period of 10 hours. This also should provide considerable time for (some) crosslinking to occur. Therefore, even if the efficiency of crosslinking does not approach $100 \%$ in the absence of exogenously added $\mathrm{CuCl}_{2}$ (as may be the case following Flag purification only), enough crosslinking is likely taking place during electrophoresis that a homomeric ring (such as the $\alpha 4 \mathrm{HMWC}$ ) can be stabilized and thus survive native PAGE.

[1] D. Panfair, A. Ramamurthy, A.R. Kusmierczyk, Alpha-ring Independent Assembly of the $20 \mathrm{~S}$ Proteasome, Sci Rep 5 (2015) 13130.

[2] G. Sun, V.E. Anderson, Prevention of artifactual protein oxidation generated during sodium dodecyl sulfate-gel electrophoresis, Electrophoresis 25 (2004) 959-965. 